\title{
Viewing China's CI Design from Shenzhen
}

\author{
Yanjun Li \\ College of Art and Design, Xi' an University of Technology, Xi' an 710054, China \\ Tel: 86-29-8206-6427Ｅ-mail: liyanjun2227478@yahoo.com.cn \\ Haozhong Yang \\ Xi' an University of Architecture and Technology, Xi' an 710055, China \\ Tel: 86-29-8262-8258Ｅ-mail: yanghaozhong0924@sina.com
}

\begin{abstract}
China is expected to start from its realities, to absorb the merits in CI of U.S. and Japan, to rely on conception and to convey enterprises' operation conception as well as culture to those individuals or organizations related to them with its integrated transimission system. As an image project, CI refers to the packaging and improvement of an enterprise's image. With our increasing knowldege about CI, we can learn more about an enterprise's conception and regulations of conduct,and accordingly, base the whole designing process on conception. Besides, an enterprise's performance should be judged according to scientific statistics instead of perceptual feelings.
\end{abstract}

Keywords: Shenzhen, CI, Transition, Conception, Brand, Opinion

Despite of its short-time existence in China, CI has experienced its unknown period, popularity and current stage as a quite common thing, which really deserves our reflections. In the preliminary stage of market economy, enterprises mainly competed with each other in desirable products. However, with increasingly fierce competition, desirable products had to give way to excellent salespersons to promote the sales of products. Nowadays, due to a lot of elements such as the constant expansion of market, the globalization of competition and the narrower gaps in quality among similar products, consumers tend to be more particular about the brand, quality, service, style and color of products. Accordingly, consumers should be satisfied not only in their practical demands but also in their aesthetic, mental and psychological demands. Therefore, company image has become an important way to improve the competitiveness of products.

Shenzhen is a pioneering field for the existence and development of CI. Ever since 1997 to 1998, CI has been widespread from Shenzhen to other parts all over China. Inspired by the success stories of Apollo, FIRS and Master Kang, many enterprises longed for CI with the hope to succeed in market competition with its magic. At that time, because most enterprises were still ignorant of CI, the CI companies in Shenzhen would earn several million yuan for a case. For example, Icon-Image Corporation, once well known for designing CI for FIRS, signed a contract with Ningbo Cigarette Factory to design its image at the price of 3,800,000 RMB. However, with the cooling of the previous upsurge, the price for CI has dropped down sharply. Then many people would ask why. Are clients more mature now or has CI failed to adapt it to the changing times?

\section{The Beginning of $\mathrm{CI}$ in China}

China's CI theory comes from Japan. Despite the 20-year delay in Japan's introducing CI compared with that in Europe and U.S. influenced by World War II, it followed U.S. to create a CI theory system with its own characteristics in 1970s. Compared with the design of Europe and U.S., Japan's design puts more emphasis on marketing-oriented visual design and the intuitionistic impressions created by logo, font and color and takes visual identity system as the core of its CI strategy. In addition, by combining its CI theory with its operation traditions, Japan came up with "Humanistic CI" and "Cultural CI", enriching elements for theoretical identification and activity identification in CI system. Meanwhile, emphasis is attached to establishing company image in the aspects of operation conception, enterprise spirit, corporate culture, institutional system and regulations of conduct and son on. By putting human beings at the center of CI, it clarifies its corporate conception and culture. It further develops its CI into CIS, in which theories about MI, BI, VI are included to emphasize the great importance they have paid to passing down its corporate culture, accumulate forces to develop enterprises and to strengthen employees' cognition and identification.

Taiwan began to introduce CI in 1967. For instance, Taiwan Plastics Company introduced CI, which became the landmark in the history of Taiwan's CI development. In addition, Weiquan Company invited Japanese designers to 
design CI for it, hence promoting Japan's influence on Taiwan in CI.

CI development in the mainland has been directly influenced by the cases of Apollo, FIRS and Master Kang. Therefore, $\mathrm{CI}$ in Japan and Taiwan has laid the theoretical basis for the development of CI here.

\section{The Current Situation and Development of $\mathrm{CI}$ in China}

$\mathrm{CI}$ is defined as taking standard fonts and trademarks to reveal corporate conception and culture in Europe and U.S.. Actually, their CI design focuses on VI, which emphasizes the standardization of visual effect, pursues the unity of designing elements and transmission media in order to employ the symbol, standardized font and color properly in the whole enterprise and therefore to convey the overall information of the whole enterprise with its visual image. However, Japan's CI is regarded as a kind of activity to clarify corporate conception and culture, which focuses on the reform of corporate conception and operation guidelines. Accordingly, in its CI design, not only visual effect, but also corporate conception and behavior should be emphasized. Efforts should be made in operation conception, enterprise orientation, value orientation and enterprise morality to standardize employees' behavior in order to promote production and create greater profit.

CI in Shenzhen focuses on VI, so do those Taiwan companies located here with MI as a complimentary enclosure. However, most CI design companies in the mainland only know some theories about MI and BI while feeling a loss when it comes to real practice. Actually, those Taiwan CI companies, just like ours, have no experience in this field, only relying on plenty of information coming from Japan's achievements in CI. In this sense, China's CI originates from Japan's CI theory system.

After the reform and opening in China, most enterprises, still exploring their operation, management and so on, had only perceptual knowledge about CI. It is in that very period that enterprises and CI design companies got in touch with this new thing. The success stories of some enterprises inspired more entrepreneurs to have their own CI designed as well as informed CI design companies of enterprises' desires for CI. As a result, CI boomed rapidly, and reached its peak in 1997 to 2000. Almost all CI design companies took the Japanese-style MI, BI and VI theory and design and tried to develop clients in a variety of ways such as holding lectures and organizing trainings and so on. In their lectures, lecturers made CI an all-purpose god by connecting it with marketing, enterprise management, staff training, quality control and institutional structure and so on.

In that period, CI companies emerged in great numbers. In order to attract more clients, most CI lecturers emphasized the difference between visual-type CI in U.S. and cultural-type CI in Japan and particularly pointed out that MI, BI, VI were created in Japan and would be the tendency of China's CI. Therefore, CI became popular, which also brought about some questions. Why should enterprises introduce CI? Is it the proper time to introduce CI? What problems can be solved by CI?

\section{The Misunderstanding and Puzzle in China's CI}

In the initial period, CI companies in Shenzhen kept wavering between design and planning, lacking in specific range and definition. From the very beginning they went to extremes, some focusing their attention to design while others to planning in which MI, BI and VI were emphasized.

Those CI design companies made progress more or less in VI design and packaging, while those companies involved in CI planning felt at a loss about the real function of CI. Their MI planning included a variety of things in a variety of ranges, such as operation conception, development strategy, target management, institutional structure, staff spirit, operation orientation, operation target and so on. As a result, clients were shocked by the thick pile of reports with words, tables and figures. In a word, neither design companies nor planning companies have realized the actual demands of Chinese enterprises. What is MI for? What can it do for enterprises? These questions are bothering CI companies, including those from Taiwan.

Actually, without any professionals in economics, management, accounting, statistics and so on, CI companies are artistic design companies in a sense. However, MI in Acer is a success because it deals with this part by itself instead of having it done by CI companies.

\section{The Deficiency and Immaturity in China's CI}

The main reason why many clients in Shenzhen aren't satisfied with those full-service CI companies lies in that these companies fail to conduct careful investigations and analysis about the market and the involved enterprise or to compare the enterprises of the same kind horizontally and vertically. Some CI companies don't conduct any analysis on image indexes when planning and designing for an enterprise, hence failing to obtain authentic and scientific basis for their decision. In most cases, CI companies don't care about the profit and the effect an enterprise will earn by introducing CI. Instead of regulating their own design thoughts and service attitudes in order to better satisfy enterprises, these CI companies keep complaining about their clients' ignorance of the current global marketing pattern and development tendency and inspire the new ideas about integration, marketing and transmission into them. In fact, they are just 
armchair strategists.

In addition, some other CI companies try to attract clients by boasting that they could save their enterprises with their so-called professional abilities. After accomplishing the design and planning for enterprises, they seem to have nothing to do with them. If anything wrong happens to MI, it is up to enterprises themselves to solve it, hence resulting in some new problems. However, regardless of hardships, Japanese CI companies have paid enough attention to these problems and have stuck to a strict, scientific and responsible attitude to their clients, which is what we should learn from them.

Meanwhile, some clients, ignorant of CI, only turn to their subjective opinions, instead of any strict and scientific data or evidence to judge the result of CI. According to most entrepreneurs, only the identification system matters, just like names for people, brands for enterprises.

As for corporate conception, some wise entrepreneurs regard it as their basis, guideline and a window for the whole society to know about them, hence preferring to deal with it by themselves. Accordingly, they are more concerned about some issues such as how to reflect their enterprises' conception and what actions or methods will help to achieve better effects and so on.

Ever since 2002, CI in Shenzhen has declined, which is attributed to, first, reduced demands for CI and, second, lowered price for CI design and planning (only 60,000 to 80,000 RMB)

\section{My Views about Current $\mathrm{CI}$ in China}

As shown in the development course of CI in Shenzhen, the most critical point lies in that CI companies mainly rely on perceptual judgment instead of a combination of scientific data analysis and the reality. Clients once spent so much money in introducing CI expecting the professional support of CI companies, only to find that what they really needed was not empty theories but solutions to practical problems. However, even up to then CI companies were struggling their way in mist, failing to satisfy the demands of clients in time.

For an enterprise, conception should be its operation principle, the basis for its competition and survival and therefore the premise of all. When establishing its corporate conception, an enterprise is expected to consider the advice of the leadership and common staff as well and then to form it by exerting creativity, which includes the significance of its existence, operation guidelines and principles. In this way, they can express their enterprise's overall image and conception with the most concise, vivid and representative schemes or words.

With more detailed division of labor, CI services should be more specific. CI companies should base their design and planning for an enterprise on its established conception and help to form wide recognition among the individuals or organizations related to this enterprise about its operation conception, corporate culture and spirit. In CI design, modern design conception and enterprise management theory should be considered to position an enterprise's individuality and characteristics in order to give prominence to the connotation of its corporate spirit. Internally, the purpose of unifying thoughts and will as well as enhancing the cohesion among individuals can be fulfilled; externally, it will help to achieve the function of enterprise identification and social recognition, and therefore to encourage enterprises to achieve their goals with their design and planning system. This standard calls more for CI companies, in whose work not only a great amount of surveys but the elaborate designs of first-class designers are required. To be more specific, during the designing process, CI companies should rely on the specific enterprise's conception and the data and material they have got in actual surveys and analysis and further to learn about its conception, rules of conduct and spiritual connotations. Only in this way will their CI designs stand the tests of time and market.

In fact, when introducing CI, some issues should be taken into consideration: first, VI should be tested in whether it will reveal an enterprise's conception and behavior in an overall and authentic way and whether it will reflect the basic idea and spirit of the enterprise's conception and behavior. If an enterprise's image is divorced from its conception and behavior, its CI will be lacking in solid base, resulting in the failure in establishing and strengthening its corporate image. Second, attention should be paid to whether an enterprise has achieved effect by specifying its operation conception. The answer of "no" reveals some problems in the process to be solved. Third, in addition to specifying conception, the transmission of conception should be tested in its systematic and strategic degree, and in whether people accept such conception and to what extent it is accepted and so on. If its conception is not accepted, surveys, analysis, research, exploration and positioning should be conducted again to test its correctness, and then to convey it after immediate amendments. Some issues, such as the recognition degree of image indexes among the staff, the design methods preferred in the target group (ephasis on font or graph), the popular color in the specific industry and some relevant regulations in its industry and so on, should be taken into consideration. When CI companies display their analysis charts based on their surveys about design method, color and other design elements to their clients and explain why to come to such a design, clients will, of course, regard them professional enough. This seems quite necessary because no client would like to pay a lot of money, only to listen to your design or planning based on your experience or feeling. Instead, they prefer to achieve their goals with the help of CI companies, which are expected to demonstrate what problems in their CI should be solved and what ways can be relied on to do that with detailed data analysis and 
scientific planning schemes.

\section{Conclusion}

CI will develop with the development in our society and culture, and of course, will vary in different areas, nations and times. Therefore, we are expected to develop our own CI by relying on our own realities as well as learning from U.S. and Japan in their CI

The value of CI (brand= product + culture) lies in its aiming at serving brands, then further promoting the branding process of enterprises and then expanding the market to earn better economic profits.

\section{References}

Cheng, Zhaohui. (2001). Graphic Design of CI. Hangzhou: China Art Academy Press.

Development of CI in Shenzhen (fourth). [online] Available: http://www.asiaci.com/.

Huang, Youzhu. (2003). The Cultural Choice of Packaging Design towards Noumenon. Packaging Engineering. 24(5): 69-71.

Wu, Weishan. Chen, Haiyan. Cai, Sanfa. (2001). Company Identification: Planning and Design. Shanghai: Shanghai People's Fine Arts Publishing House.

Zhang, Chenglai. Han, Xiang. (2002). The Design of CIS. Hebei: Hebei Fine Arts Publishing House. 